\title{
Spectroscopy of an AdS Reissner-Nordstrøm black hole
}

\author{
Claudio Dappiaggi ${ }^{1,2, a}$, Simona Raschi ${ }^{1}$, \\ ${ }^{1}$ Dipartimento di Fisica Nucleare e Teorica, Università di Pavia via A.Bassi 6 I-27100 \\ Pavia, Italy. \\ ${ }^{2}$ Istituto Nazionale di Fisica Nucleare Sezione di Pavia, via A.Bassi 6 I-27100 Pavia, \\ Italy. \\ ${ }^{a}$ claudio.dappiaggi@pv.infn.it
}

\begin{abstract}
In the framework of black hole spectroscopy, we extend the results obtained for a charged black hole in an asymptotically flat spacetime to the scenario with non vanishing negative cosmological constant. In particular, exploiting Hamiltonian techniques, we construct the area spectrum for an AdS Reissner-Nordstrøm black hole.
\end{abstract}




\section{Introduction}

The road leading to an explicit formulation of a full quantum theory of gravity necessarily requires a complete comprehension of the role of extreme gravitating objects such as black holes. In particular a key question concerns the characterization and the construction of their Fock space of states in a quantum version of general relativity. An answer would grant us the chance to study the spectrum of operators acting on the Fock space and, consequently, the quantum numbers characterizing the expectation values. Furthermore, this project would ultimately put to test the long standing conjecture according to which a discrete spectrum is associated to the area operator of the event horizon.

In this connection, a landmark has been set by Bekenstein (see [1 and 2]) who suggested that the area of the event horizon of a black hole behaves as an adiabatic invariant of the associated dynamical system. With this hypothesis and assuming that the standard rules of quantum mechanics could also be applied in a strong gravitational field, it is possible to appeal to the so-called Ehrenfest principle. It grants that a quantum operator with a discrete spectrum corresponds to any classical "adiabatic" observable on a phase space. The reasonableness of the above assumption has been addressed by several authors and, in particular, it holds for a neutral non rotating black holes 3, 4. The natural subsequent step consists of conjecturing that the most general form for the area spectrum is:

$$
A=n a_{0},
$$

where $n$ is an integer number and $a_{0}$ is a constant proportional to $l_{p}^{2}$, the square of the Planck length. Under the same hypothesis, this result have been generalized to charged black holes, such as Reissner-Nordstrøm, with one notable exception: the role of adiabatic invariant is not played by the area $A(M, q)$ depending on the mass $M$ and the charge $q$ but by the difference between $A(M, q)$ and the area $A_{\text {ext }}(q)$ of the black hole in the extremal classical configuration [5].

The only setback in the above construction lies in the heuristic nature of Bekenstein results which, thus, cannot provide any precise mean to rigorously justify the shape of the area spectrum and, in particular, to find an exact value for the constant $a_{0}$ in (1). The are two notables approaches for making more precise and predictable the above arguments; the first has been proposed by Bekenstein himself [1, 2] and it is based on an axiomatic point of view according to which one constructs a closed and linear algebra of observables for the black hole Fock space such that the spectrum can be directly inferred from the properties of the algebra itself. Within this framework, (11) is confirmed on a firmer ground though the specific value for the constant $a_{0}$ cannot be derived. The second approach instead concentrates on a Hamiltonian formulation [5] i.e., by exploiting a generalized Birkhoff theorem, the dynamic of a spherically symmetric background, can be described on a "reduced phase space" where the main variables are diffeomorphisms invariant physical observables quantities, such as the black hole mass and charge, and their associated conjugate momenta. Within this framework the rationale is to select a suitable canoni- 
cal transformation of coordinates for the phase space in such a way that the Hamiltonian for the dynamical system is similar to that of a harmonic oscillator. A rather direct quantization procedure for the Hamiltonian itself and, when necessary, for the charge sector, allows to directly retrieve the area spectrum. The advantage of this scheme over the axiomatic one lies in the absence of any a priori assumption either on the shape of the spectrum or on the existence of a particular algebra of operators. Furthermore the results confirm Bekenstein suggestions and a specific value for $a_{0}$ can be directly inferred; it is also possible to show that ultimately both methods, the axiomatic and the reduced phase space, are completely equivalent, either for charged either for neutral, non rotating black holes [6].

The phase space approach has been applied to a wide class of specific black holes, the most notables being the Schwarzschild (see also [7), the ReissnerNordstrøm [5] and the Kerr black holes [8, 9] though, in all these scenario, a condition of asymptotic flatness, has been imposed. It is thus natural to ask ourselves whether it is possible to generalize the above results when a different class of spacetimes is chosen. Here we consider asymptotically AdS black holes, though we will not refer to the neutral non rotating case which can be seen as a particular subcase of [15] and of [16]. On the opposite we concentrate on the AdS Reissner-Nordstrøm case, we construct the area spectrum and we test if it is still equispaced even when the charged sector is taken into account. In other words, we consider what is the effect of the cosmological constant on the eigenvalues of the area operator.

Beside testing Bekenstein ideas in a broader setting, there are wider motivations to address this question since asymptotically AdS backgrounds play a central role in current research. In particular an answer to the above query should be seen as a starting point to test the results of black hole spectroscopy from the point of view of the AdS/CFT correspondence [10 and to compare them to other approaches to area quantization [11, in particular that of quasi normal modes [12, 13] where a comparison with Bekenstein approach is still rather elusive at least in an asymptotically flat black hole background (see 14] for a recent exhaustive review).

The outline of the paper is the following: in section 2 we review the reduced phase space approach and the scheme of quantization. We will also discuss this method in the context of an asymptotically flat Reissner-Nordstrøm black hole. In section 3 we deal instead with the AdS counterpart explicitly constructing the spectrum and commenting on the result. In section 4 we draw some conclusion and we propose some possible future lines of research. Eventually, in the appendix, we review the axiomatic approach in connection with the method discussed in sections 2 and 3. 


\section{The reduced phase space approach}

As we have outlined in the introduction, the remarkable arguments put forward by Bekenstein, in order to calculate the spectrum of the event horizon area for a black hole, are heuristics and they are considered as the starting point to set the black hole spectroscopy in a more rigorous framework which is here introduced and reviewed.

\subsection{Construction of the area spectrum}

The rigorous approach to black hole spectroscopy, we now discuss, is often known as reduced phase space formulation [5, 15, 16, 17, 18. Since our aim is to ultimately apply this specific method to a charged spherically symmetric non rotating AdS black hole, we start from a rather general framework, specializing the discussion in the next section to the asymptotically flat Reissner-Nordstrøm black hole.

The starting point is a four dimensional Lorentzian smooth manifold $\mathcal{M}^{4}$ and the Einstein-Maxwell action [19]

$$
S=\int_{\mathcal{M}^{4}} d^{4} x \sqrt{|g|}\left(\frac{R}{16 \pi}-\frac{1}{4 \pi} F^{\mu \nu} F_{\mu \nu}\right),
$$

where $F_{\mu \nu}=A_{[\mu ; \nu]}$, being $A_{\mu}$ the $U(1)$ electromagnetic potential. If we introduce the usual coordinate system $(x, t, \theta, \varphi)$, a generic spherically symmetric metric can be written as

$$
d s^{2}=g_{\alpha \beta} d x^{\alpha} d x^{\beta}+r^{2}\left(x_{\alpha}\right) d^{2} \Omega(\theta, \varphi),
$$

where $d^{2} \Omega(\theta, \varphi)$ is the solid angle element and where the strictly positive valued function $r\left(x_{\alpha}\right)$, the radius of the sphere and the metric $g_{\alpha \beta}$ depend only upon the coordinates $x_{\alpha} \doteq\{t, x\}$ spanning a two dimensional spacetime $\mathcal{M}^{2}$. Assuming that also $A_{\mu}$ is spherically symmetric, a direct substitution of (3) in (2) provides, upon integration of the angular coordinates and under the rescaling $r \rightarrow \sqrt{2} r$, the dimensionally reduced action (c.f. section 2 in [20] - see also [21] and [22])

$$
S=\int_{\mathcal{M}^{2}} d^{2} x \sqrt{|g|}\left[\frac{1}{2}\left(\frac{g_{\alpha \beta}}{2} \partial^{\alpha} r \partial^{\beta} r+1+\frac{r^{2}}{2} R\left(g_{\alpha \beta}\right)\right)-\frac{r^{2}}{4} F^{(2) \alpha \beta} F_{\alpha \beta}^{(2)}\right]
$$

where $R\left(g_{\alpha \beta}\right)$ is the scalar curvature associated with the two dimensional metric $g_{\alpha \beta}$ and $F_{\alpha \beta}^{(2)}$ is the two dimensional electromagnetic field strength. For our purposes, dimensional reduction plays a key role since it allows us to perform a Hamiltonian analysis of the spherically symmetric Einstein-Maxwell action along the lines of [23. In this last cited paper a more general class of dilatonic actions have been taken into account namely:

$$
S^{\prime}=\int_{\mathcal{M}^{2}} d^{2} x \sqrt{|g|}\left[\frac{1}{2}\left(\frac{g_{\alpha \beta}}{2} \partial^{\alpha} \psi \partial^{\beta} \psi+V(\psi)+D(\psi) R\right)-\frac{W(\psi)}{4} F^{\alpha \beta} F_{\alpha \beta}\right]
$$


where $R$ is the scalar curvature, $\psi$ plays the role of the dilaton field, $F_{\alpha \beta}$ is the electromagnetic field strength and where $V(\psi), D(\psi)$ and $W(\psi)$ are generic functions. Whenever $D(\psi)$ is at least a differentiable function of $\psi$ such that $D(\psi) \neq 0$ and $\frac{d D(\psi)}{d \psi} \neq 0$, it is possible to cancel the kinetic term by means of a suitable field redefinition [20]:

$$
\bar{g}_{\alpha \beta}=\Omega^{2}(\psi) g_{\alpha \beta}, \quad \phi=D(\psi) .
$$

Here $\Omega^{2}(\psi)$ is a conformal rescaling which, up to an irrelevant constant, is set by

$$
\Omega^{2}(\psi)=\exp \left(\frac{1}{2} \int\left(\frac{d D(\psi)}{d \psi}\right)^{-1} d \psi\right) .
$$

According to (6), (5) becomes

$$
S^{\prime}=\int_{\mathcal{M}^{2}} d^{2} x \sqrt{|\bar{g}|}\left[\frac{1}{2}(\phi R+V(\phi))-\frac{W(\phi)}{4} F^{\alpha \beta} F_{\alpha \beta}\right] .
$$

An important property of this action has been discussed in 24 and in 25] where a Birkhoff-like theorem has been proved. In detail, it is always possible to find a suitable local coordinate frame such that the metric arising from a solution of the Euler-Lagrange equations of (8) is static and it depends only upon diffeomorphism invariant parameters. If we introduce a suitable time coordinate $\tau$ and the spatial coordinate $\sigma \equiv \phi$, the most general solution can be written as

$$
\begin{gathered}
d s^{2}=-f(\sigma ; C, q) d \tau^{2}+f^{-1}(\sigma ; C, q) d \sigma^{2}, \\
f(\sigma ; C, q)=-C+j(\sigma)-q^{2} k(\sigma), \\
F_{\alpha \beta}=\frac{q}{W(\sigma)} \epsilon_{\alpha \beta},
\end{gathered}
$$

where $C, q$ are two constants of integration which are respectively related to the $A D M$ mass and to the $U(1)$ electric charge, as we will clarify when we will deal with the Hamiltonian formulation. The functions $j(\sigma), k(\sigma)$ instead are implicitly defined from the equations

$$
V(\sigma)=\frac{d j(\sigma)}{d \sigma}, \quad W^{-1}(\sigma)=\frac{d k(\sigma)}{d \sigma} .
$$

We can now discuss the above mentioned Hamiltonian analysis; as a starting point we assume that $\mathcal{M}^{2}$ is locally $\mathbb{R} \times \mathcal{C}$ where $\mathcal{C}$ is a one dimensional spatial manifold, not necessarily closed. A generic metric can be written as

$$
d s^{2}=e^{2 \rho}\left[-N_{1}^{2} d t^{2}+\left(d x+N_{2}^{2} d t\right)^{2}\right],
$$

where $x$ now is a local coordinate for $\mathcal{C}$ and $\rho, N_{1}, N_{2}$ are generic functions of $(t, x)$. Plugging this metric in (8) and slavishly repeating the analysis of [24, 
we find that the momenta associated to $N_{1}, N_{2}$ and to $A_{0}$, vanish; consequently these fields play the role of Lagrange multipliers and, besides the dilaton $\phi$, only $\rho$ and $A_{1}$ are dynamical degrees of freedom. If we introduce the conjugate momenta $\Pi_{\rho}, \Pi_{\phi}, \Pi_{A_{1}}$, the full Hamiltonian is the sum of two components, the first is the canonical Hamiltonian $H_{\text {can }}$. whereas the second is a surface term needed whenever $\mathcal{C}$ is non compact in order for the Hamilton equations to be fully consistent with the Euler-Lagrange equations i.e.:

$$
\begin{gathered}
\mathcal{F}=\rho^{\prime} \Pi_{\rho}+\phi^{\prime} \Pi_{\phi}-\Pi_{\rho}^{\prime} \sim 0, \quad \mathcal{I}=-\Pi_{A_{1}}^{\prime} \sim 0 \\
\mathcal{G}=\phi^{\prime \prime}-2 \phi^{\prime} \rho^{\prime}-2 \Pi_{\phi} \Pi_{\rho}-e^{2 \rho} V(\phi)+\frac{e^{2 \rho}}{W(\phi)} \Pi_{A_{1}}^{2} \sim 0, \\
H=H_{\text {can. }}+H_{\text {surf. }}=\int d x\left[N_{1} \frac{\mathcal{G}}{2}+N_{2} \mathcal{F}+A_{0} \mathcal{I}\right]+H_{\text {sur. }},
\end{gathered}
$$

where $\mathcal{F}, \mathcal{G}$ generate spacetime diffeomorphisms and $\mathcal{I}$ enforces the $U(1)$ gauge transformation. These are secondary constraints and the symbol $\sim 0$ implies that they are weakly vanishing in the Dirac sense (c.f. chapter 12 of [26]). Consequently we are dealing with a dynamical system with six first class constraints, three primary $N_{1}, N_{2}, A_{0}$ and three secondary $\mathcal{F}, \mathcal{G}, \mathcal{I}$; according to 24 and to [27, the latter can be solved as:

$$
\Pi_{\rho}=Q[C, q, \rho, \phi], \quad \Pi_{\phi}=\frac{g[q, \rho, \phi]}{4 Q[C, q, \rho, \phi]}, \quad \Pi_{A_{1}}=q,
$$

where $C, q$ are two constants of integration which turn out to be the same as those introduced in (10) i.e. they are respectively (proportional to) the ADM mass and the electric charge. Moreover a straightforward counting shows that, to each point in $\mathcal{M}^{2}$, it is associated a six dimensional phase space and the six above mentioned first class constraints, thus there are no propagating modes in the dynamical system described by (8).

The next step lies in the key observation that $C, q$ have vanishing Poisson brackets with all the constraints and between them; consequently they can be written in terms of the original phase space coordinates as

$$
C=e^{-2 \rho}\left[\Pi_{\rho}^{2}-\left(\phi^{\prime}\right)^{2}\right]+j(\phi)-k(\phi) \Pi_{A_{1}}^{2}, \quad q=\Pi_{A_{1}},
$$

where, as before, $\frac{d j(\phi)}{d \phi}=V(\phi)$ and $\frac{d k(\phi)}{d \phi}=W^{-1}(\phi)$.

Thus, from a direct inspection of (12) and of (13), it appears that both $C$ and $q$ are independent from the spatial coordinate on $\mathcal{C}$, but, more importantly, they are also the the unique independent physical observables in the Dirac sense [24].

Let us now introduce the canonical momenta associated to $C, q$ namely $\Pi_{C}, \Pi_{q}$ referring for an explicit expression and a discussion about their specific properties to section IV and VI in 24. We simply remark that, according to the analysis in [28, $\Pi_{q}$ is related to the asymptotic choice for the $U(1)$ gauge whereas, if we consider an evolution between two different instants of time, $\Pi_{C}$, 
at infinity, can be physically interpreted as the time separation. Furthermore the following key relationship holds [5, 24]:

$$
\delta \Pi_{q}=\frac{\Phi}{2} \delta \Pi_{C}+\delta \lambda,
$$

where $\delta$ refers to a variation under a change on the boundary conditions and where $\Phi$ is the electrostatic potential calculated at the boundary under consideration. If we take into account the above remarks and the absence of propagating modes, we can switch from the six dimensional phase space generated by $\left(\rho, \phi, A_{1}, \Pi_{\rho}, \Pi_{\phi}, \Pi_{A_{1}}\right)$ to a reduced phase space $\Gamma \sim \mathbb{R}^{4}$ generated by $\left(M, q, \Pi_{M}, \Pi_{q}\right)$.

In order to complete the analysis of the system ruled by (8), the last step consists of discussing $H_{\text {surf. }}$ in (14) i.e. we need to impose suitable boundary conditions on the phase space variables; we follow 24] and mainly 23] where this issue is dealt with in detail for charged black holes arising in two dimensional dilatonic gravity. Let us nonetheless briefly summarize some key points and results: by direct substitution of (16) and of the explicit expression for $\Pi_{\rho}$, $H_{\text {can }}$ in (14) becomes

$$
H_{\text {can }}=\int_{\mathcal{C}} d x\left[\frac{\dot{\phi}}{\phi} \mathcal{F}-\frac{1}{2}\left(\frac{\sigma e^{2 \rho}}{\phi^{\prime}} C^{\prime}-A_{0} q^{\prime}\right)\right] .
$$

For any metric whose expression approaches asymptotically (9) in such a way that, at spatial infinity, $\dot{\phi} \rightarrow 0$ and $\frac{\sigma e^{2 \rho}}{\phi^{\prime}} \rightarrow 1$, the surface term can thus be chosen as

$$
H_{\text {sur. }}=\int_{\mathcal{C}} d x \frac{1}{2}\left(\frac{\sigma e^{2 \rho}}{\phi^{\prime}} C\right)^{\prime}+\left(A_{0} q\right)^{\prime},
$$

where we can identify in the first term the ADM Hamiltonian $H_{A D M}$ and, consequently the ADM mass $M=\frac{C}{2}$. In spite of this result, from now on, we will switch from the coordinates $\left(C, q, \Pi_{C}, \Pi_{q}\right)$ for the phase space $\Gamma$ to the fully equivalent coordinates $\left(M, \Pi_{M}, q, \Pi_{q}\right)$. Within this framework it is possible to write the action (5) as a function on the reduced phase space i.e.

$$
I=\int d t\left[\Pi_{M} \dot{M}+\Pi_{q} \dot{q}-H(M, q)\right],
$$

where, according to the previous discussion, the Hamiltonian is independent from the momenta.

Let us now slightly specialize our analysis to a specific scenario where (9) describes a black hole background and, thus, necessarily it exists at least a value for $\sigma$, say $\bar{\sigma}$, such that $f(\bar{\sigma}, M, q)=0$. Within this framework, the action (18) represents the starting point in [5, 17, 18, to explicitly construct the black hole entropy spectrum and consequently the area spectrum since, as we will emphasize later, we consider only black holes where the Bekenstein entropy to area ratio holds. The first step in the analysis involves a Wick rotation from 
the Lorentzian to the Euclidean metric. From a semiclassical thermodynamical analysis, we can infer that the mass momentum becomes periodic i.e. $\Pi_{M}$ must be identified with $\Pi_{M}+\frac{1}{T_{H}(M, q)}$ where $T_{H}(M, q)$ is the Hawking temperature. Thus the phase space $\Gamma$ becomes topologically equivalent to $\mathbb{R}^{3} \times S^{1}$ and the circle, parametrized by $\Pi_{M}$, can be unwrapped by means of a coordinate transformation $\left(M, \Pi_{M}, q, \Pi_{q}\right) \rightarrow\left(X, \Pi_{X}, q, \Pi_{q}^{\prime}\right)$ :

$$
\begin{gathered}
\Pi_{X}=\sqrt{\frac{B(M, q)}{\pi}} \sin \left(2 \pi \Pi_{M} T_{H}(M, q)\right), \quad \Pi_{q}^{\prime}=\Pi_{q}^{\prime}\left(M, \Pi_{M}, q, \Pi_{q}\right), \\
X=\sqrt{\frac{B(M, q)}{\pi}} \cos \left(2 \pi \Pi_{M} T_{H}(M, q)\right),
\end{gathered}
$$

where both $B(M, q)$ and $\Pi_{q}^{\prime}\left(M, \Pi_{M}, q, \Pi_{q}\right)$ are functions which are set by the request that the above change of coordinates is canonical i.e.

$$
\begin{aligned}
\Pi_{X} \delta X & +\Pi_{q} \delta q=\Pi_{M}\left(T_{H}(M, q) \frac{\partial B(M, q)}{\partial M}\right) \delta M+ \\
& +\left(\Pi_{q}+\Pi_{M} T_{H}(M, q) \frac{\partial B(M, q)}{\partial q}\right) \delta q .
\end{aligned}
$$

This implies:

$$
\frac{\partial B(M, q)}{\partial M}=T_{H}^{-1}(M, q), \quad \Pi_{q}^{\prime}=\Pi_{q}-T_{H}(M, q) \frac{\partial B(M, q)}{\partial q},
$$

where the first equality, together with the first law of thermodynamics for black holes, grants us that $\frac{\partial B(M, q)}{\partial M}=\frac{\partial S(M, q)}{\partial M}$ or, equivalently, upon integration

$$
B(M, q)=S(M, q)+F(q) .
$$

The function $F(q)$ is an a priori arbitrary function though it is commonly chosen as $-S_{0}(q)$, the minimum of the entropy $S(M, q)$ in terms of $M, q$. The reasons behind this prescription are several, the most notable lying in the coordinate transformations (19) and (20) which grant us that the region $\left(M, \Pi_{M}\right) \sim S^{1} \times$ $\mathbb{R} \subset \Gamma$ is mapped into $\left(X, \Pi_{X}\right) \sim \mathbb{R}^{2}-\mathbb{D}$ where $\mathbb{D}$ is a disk of radius $S_{0}(q)+$ $F(q) \geq 0$. Thus $F(q)=-S_{0}(q)$ grants us that the subspace $\left(X, \Pi_{X}\right)$ of the phase space with coordinates $\left(X, \Pi_{X}, q, \Pi_{q}^{\prime}\right)$ is $\mathbb{R}^{2}$. As shown in [5], the above choice also uniquely determine $\Pi_{q}^{\prime}$ as

$$
\Pi_{q}^{\prime}=\Pi_{q}+\Phi \Pi_{M}+\Pi_{M} T_{H}(M, q) \frac{d S_{0}(q)}{d q},
$$

where $\Phi$ is the electrostatic potential at the boundary of the region of spacetime under consideration.

Considering the phase space variables $\left(X, \Pi_{X}, q, \Pi_{q}^{\prime}\right)$ as observables on a suitable Hilbert space of square integrable functions in such a way that they 
satisfy the canonical commutation relations $\left[\hat{X}, \hat{\Pi}_{X}\right]=\left[\hat{q}, \hat{\Pi}_{q}\right]=i$, we can directly obtain the area spectrum. By direct inspection of (19), (20) and of (21) we end up with:

$$
S(M, q)-S_{0}(q)=\pi\left(\hat{X}^{2}+\hat{\Pi}_{X}^{2}\right) .
$$

The right hand side is a self-adjoint operator on the above mentioned Hilbert space and it can be straightforwardly quantized as in ordinary quantum mechanics:

$$
S(M, q)-S_{0}(q)=2 \pi\left(n+\frac{1}{2}\right) . \quad n \in \mathbb{N}
$$

In order to complete our task, we need also to quantize the electromagnetic sector and this can be achieved following 5 where, starting from (17), it has been shown that the phase space points $\left(q, \Pi_{q}^{\prime}\right)$ and $\left(q, \Pi_{q}+2 \pi \frac{n_{1}}{e}+n_{2} \frac{d S_{0}(q)}{d q}\right)$ $\left(n_{1}, n_{2} \in \mathbb{N}\right)$ must be identified i.e. the subspace spanned by $\left(q, \Pi_{q}^{\prime}\right) \sim S^{1} \times \mathbb{R}$. If we consider a momenta representation for the charge operator i.e. $\hat{q}=i \frac{\partial}{\partial \Pi_{q}}$, then the wavefunction for a charge eigenstate is proportional to $e^{i q \Pi_{q}}$ which must be single-valued. Considering the above periodicity for the $q$-variable, the request of singlevaluedeness implies that

$$
n_{1} \frac{q}{e}+n_{2} \frac{q}{2 \pi} \frac{d S_{0}(q)}{d q} \in \mathbb{N}
$$

which grants us the quantization rules:

$$
q=m e, \quad \frac{q}{2 \pi} \frac{d S_{0}(q)}{d q}=p .
$$

It is worthwhile underlining that the above equations are not independent and they ultimately provide a constraint on the possible values of $e$ in terms of $p$ and $m$ [5]. Thus, upon selecting a specific black hole background and consequently a specific expression for $S_{0}(q)$, (23) together with (24) provides a quantized expression for the entropy. At this stage, some comments are in due course:

- if we consider scenarios where the Bekenstein entropy to area ratio holds, (23), together with (24), automatically provides an area spectrum,

- $S_{0}(q)$ is the minimum of $S(M, q)$ and thus, if the relation $S(M, q)=\frac{A(M, q)}{4}$ holds, $A_{0}(q)$ represents the event horizon area for the black hole in the extremal configuration; a similar assertion cannot be stated for $S_{0}(q)$ since, in this specific scenario, such as for example the extremal ReissnerNordstrøm black hole, the classical entropy is vanishing and thus the Bekenstein formula does not seem to hold. This issue is nonetheless still under debate mainly due to apparent discrepancy between the results achieved from thermodynamical arguments and those inferred from a statistical counting of (extremal) black hole degrees of freedom in string theory. We refer to 29] and references therein for a recent discussion on this specific problem. 


\subsection{The spectrum of an asymptotically flat Reissner-Nord strøm black hole}

The approach discussed in the previous section can be applied to several scenarios though the most natural and one of the first analyzed is the asymptotically flat Reissner-Nordstrøm black hole. The starting point is (44) which appears to be a rather specific subcase of (5) where the radius $r$ of the sphere plays the role of the dilaton $\psi$ and where $V(r)=1, D(r)=\frac{r^{2}}{2}$ and $W(r)=r^{2}$. Consequently, solving (7) as $\Omega^{2}(r)=r=\sqrt{2 \phi}$, we can recast (4) as

$$
S=\int_{\mathcal{M}^{2}} d^{2} x \sqrt{|\bar{g}|}\left[\frac{1}{2}\left(\phi R\left(g_{\alpha \beta}\right)+\sqrt{\frac{1}{2 \phi}}\right)-(2 \phi)^{\frac{3}{2}} F^{(2) \alpha \beta} F_{\alpha \beta}^{(2)}\right] .
$$

A solution for the Euler Lagrange equation for this action can be directly inferred from (9), (10) and (11); since, in this specific setting, $2 \sigma=r^{2}$, we end up with $j(\sigma)=\sqrt{2 \sigma}$ and $k(\sigma)=-(2 \sigma)^{-\frac{1}{2}}$ i.e., switching back from $\sigma$ to the $r$ coordinate,

$$
\begin{gathered}
F_{\alpha \beta}=-\frac{q}{r^{2}} \epsilon_{\alpha \beta}, \\
d s^{2}=-r\left[1-\frac{C}{r}+\frac{q^{2}}{r^{2}}\right] d \tau^{2}+r\left[1-\frac{C}{r}+\frac{q^{2}}{r^{2}}\right]^{-1} d r^{2},
\end{gathered}
$$

which, bearing in mind the conformal rescaling (6) and plugging (27) in (3), is the Reissner-Nordstrøm metric with $C$ identified with twice the black hole mass $M$. It is straightforward to realize, starting from (27), that the analysis in the previous section can be applied to this specific scenario and thus we are entitled to construct the entropy/area spectrum. The unique step consists in remembering that the radii of the inner and outer event horizons are

$$
r_{ \pm}=M \pm \sqrt{M^{2}-q^{2}} .
$$

Thus the entropy is $S(M, q)=\pi r_{+}^{2}$ whereas the extremal configuration is achieved whenever $M=q$ (i.e. $r_{+}=r_{-}=q$ ) which implies either that $S_{0}(q)=\pi q^{2}$ either that (24) becomes

$$
q=m e, \quad q^{2}=p,
$$

or equivalently $e^{2}=\frac{p}{m^{2}}$. This quantization rule for the charge can be set in (23), eventually finding the area/entropy spectrum

$$
S=\frac{A}{4 G}=2 \pi n+\pi(p+1) .
$$

Here we have restored the explicit dependence on the Newton constant $G$ since, bearing in mind that the adiabatic invariant is $A(M, q)-A_{\text {ext. }}(q)$, a direct inspection of (28) shows that the value of $a_{0}$ in (11) can be set to $8 \pi l_{p}^{2}$. An interesting consequence of this result arises if we bear in mind that the vacuum 
classical configuration of a Reissner-Nordstrøm black hole corresponds to the extremal configuration with area $A_{\text {ext }}=\pi q^{2}=\pi p$. If we interpret the quantum number $n$ in (28) as labelling the excited levels over the vacuum $n=0$, the classical lower bound is never reached due to the presence of an additional term $\pi$ which can be interpreted as a symptom of vacuum fluctuations. Thus we can conclude that the extremal configuration does not lie in the spectrum constructed with this specific quantization technique and this feature will be common also when we will deal, in the next section, with a non vanishing cosmological constant scenario.

\section{The Reissner-Nordstrøm AdS black hole}

Let us now address the main question of this paper namely if we can extend the above results for a charged non rotating asymptotically flat black hole to the asymptotically AdS counterpart. Adopting the same notations and conventions from the previous sections, the starting point is the Einstein-Maxwell action with a cosmological term i.e. [19]

$$
S=\int_{\mathcal{M}^{4}} d^{4} x \sqrt{|g|}\left(\frac{R-2 \Lambda}{16 \pi}-\frac{F_{\mu \nu} F^{\mu \nu}}{4}\right),
$$

where $\Lambda<0$ is the cosmological constant. If we look for spherically symmetric solutions for the Euler-Lagrange equations, we can introduce a coordinate frame $\left(x_{0}, x_{1}, \theta, \varphi\right)$ and we can write the metric as

$$
d s^{2}=g_{\alpha \beta} d x^{\alpha} d x^{\beta}+r^{2}\left(x_{\alpha}\right) d \Omega^{2}(\theta, \varphi),
$$

where $g_{\alpha \beta}$ is a two dimensional metric depending upon the coordinates $x_{\alpha} \doteq$ $\left\{x_{0}, x_{1}\right\}$ spanning a two dimensional submanifold $\mathcal{M}^{2} \subset \mathcal{M}^{4}$.

Assuming that also the $U(1)$ vector potential $A_{\mu}$ is spherically symmetric, we look for the dimensionally reduced action plugging the above metric in (29). A straightforward calculation ends up with

$S=\int_{\mathcal{M}^{2}} d^{2} x \sqrt{|g|}\left[\left(\frac{g_{\alpha \beta}}{4} \partial^{\alpha} r \partial^{\beta} r+\frac{1}{2}+\frac{r^{2}}{4}\left(R\left(g_{\alpha \beta}\right)-2 \Lambda\right)\right)-\frac{r^{2}}{4} F^{(2) \alpha \beta} F_{\alpha \beta}^{(2)}\right]$,

This is again a special case of (5) with $r\left(x_{\alpha}\right)$ playing the role of the dilaton field, $D(r)=\frac{r^{2}}{2}, W(r)=r^{2}$, but $V(r)=1-\Lambda r^{2}$. Thus we can exploit (6) and (17) redefining the dilaton as $\phi=\frac{r^{2}}{2}$ and introducing the conformal factor $\Omega^{2}(\phi)=r=\sqrt{2 \phi}$; consequently the above action is transformed in

$$
S=\int_{\mathcal{M}^{2}} d^{2} x \sqrt{|\bar{g}|}\left[\frac{1}{2}\left(\phi R\left(g_{\alpha \beta}\right)+\sqrt{\frac{1}{2 \phi}}-\Lambda \sqrt{2 \phi}\right)-(2 \phi)^{\frac{3}{2}} F^{(2) \alpha \beta} F_{\alpha \beta}^{(2)}\right] .
$$

A solution for the Euler-Lagrange equation for this action can be inferred directly from (91), (10) and (11), namely, bearing in mind that the role of the 
spatial coordinate $\sigma$ is played by $\phi$, we calculate $k(\sigma)=-(2 \sigma)^{-\frac{1}{2}}$ whereas $j(\sigma)=\sqrt{2 \sigma}-\frac{\Lambda}{3}(2 \sigma)^{\frac{3}{2}}$. Switching back to the $r$-coordinate by means of $r^{2}=2 \sigma$, we end up with

$$
\begin{gathered}
F_{\alpha \beta}=-\frac{q}{r^{2}} \epsilon_{\alpha \beta} \\
d s^{2}=-r\left[1-\frac{C}{r}+\frac{q^{2}}{r^{2}}-\frac{\Lambda}{3} r^{2}\right] d \tau^{2}+r\left[1-\frac{C}{r}+\frac{q^{2}}{r^{2}}-\frac{\Lambda}{3} r^{2}\right]^{-1} d r^{2},
\end{gathered}
$$

which is, up to the conformal rescaling (6), the AdS Reissner-Nordstrøm metric with $C$ identified with twice the black hole mass $M$ (see [30] and sections 24.2, 24.4 in 31]).

As it is shown in the previous discussion, the main effect of the cosmological constant from the point of view of dimensionally reduced gravity concerns the variation of the potential $V(r)$ which is no more a constant, but it acquires a term directly dependant on the $r$-variable. Nonetheless, from the point of view of the Hamiltonian approach to black hole spectroscopy, we can still repeat from scratch the analysis discussed in section 2.1. Thus we can introduce a generic two dimensional metric $d s^{2}=e^{2 \rho}\left[-N_{1} d t^{2}+\left(d x+N_{2} d t\right)^{2}\right]$ and we can repeat the same analysis ending up with the Hamiltonian (14).

The only subtlety lies in the discussion of the boundary term $H_{\text {surf }}$; within this respect it is interesting to notice that the analysis in [24], summarized in section 2.1 do not require a priori the asymptotical flatness of the metric, but only that it tends at least asymptotically to (9), thus it can be applied to an AdS background. We will not discuss this issue in details in this paper; suffice to say that a more detailed analysis of the Hamiltonian dynamic for a charged AdS black hole has been presented in 32] (c.f. sections II e IV) though without the language of dilatonic gravity. In particular it is shown that, also for an AdS Reissner-Nordstrøm black hole, it is possible to construct a reduced phase space depending only upon the black hole mass $M$, the $U(1)$ electric charge $q$ and the conjugate momenta respectively $\Pi_{M}$ and $\Pi_{q}$. We Wickrotate the Lorentzian time to the Euclidean counterpart in such a way that, in order to avoid a conical singularity in the metric, the latter must be periodic with period $T_{H}^{-1}(M, q)=4 \pi r_{+}\left(1-\frac{q^{2}}{r_{+}^{2}}-\frac{\Lambda r_{+}^{2}}{2}\right)^{-1}$, being $r_{+}$the outer horizon radius of the AdS Reissner-Nordstrøm black hole. The same result translates to $\Pi_{M}$ i.e. $\Pi_{M} \sim \Pi_{M}+T_{H}^{-1}(M, q)$; slavishly repeating the construction from the previous section, it is now possible to demonstrate that the Hamiltonian ultimately depends only upon the four mentioned reduced phase space variables and on the boundary conditions, thus it can be written as (18).

Eventually we are also entitled to consider in the reduced phase space $\Gamma$ the canonical transformations of variables (19) and (20). The final result mimics that of an asymptotically flat Reissner-Nordstrøm black hole i.e.

$$
S(M, q)-S_{0}(q)=\pi\left(\hat{X}^{2}+\hat{\Pi}_{X}^{2}\right),
$$

where we have interpreted the phase space variables as operators on a suitable 
Hilbert space. Consequently we end up with the following entropy spectrum:

$$
S(M, q)-S_{0}(q)=2 \pi\left(n+\frac{1}{2}\right) .
$$

The real difference in comparison with the scenario considered in the previous section lies in the expression for $S_{0}(q)$ and in the quantization for the electromagnetic sector. As discussed previously, $S_{0}(q)$ stands, classically, for the entropy of an extremal black hole i.e.

$$
S_{0}(q)=\pi r_{e x t}^{2}=\pi\left[\frac{1-\sqrt{1-4 q^{2} \Lambda}}{2 \Lambda}\right],
$$

whereas the charge can be quantized by means of (24) i.e.

$$
q=m e, \quad \frac{q^{2}}{\sqrt{1-4 q^{2} \Lambda}}=p .
$$

As stated in section 2.1 these two quantization conditions must be simultaneously satisfied providing a constraint for the value of $e$ namely

$$
e^{2}=\frac{-2 m^{2} p^{2} \Lambda+\sqrt{8 m^{4} \Lambda^{2} p^{4}+p^{2} m^{4}}}{m^{4}} .
$$

By direct substitution of (36) in (35), we find

$$
S_{0}(q)=\frac{\pi}{2 \Lambda}+\pi p\left(1+\sqrt{1+\frac{1}{4 \Lambda^{2} p^{2}}}\right),
$$

which, together with (34), provides the full AdS Reissner-Nordstrøm spectrum:

$$
S(M, q)=2 \pi n+\pi p\left(1+\sqrt{1+\frac{1}{4 \Lambda^{2} p^{2}}}\right)+\pi\left(1+\frac{1}{2 \Lambda}\right) .
$$

At this stage, a few remarks are in due course:

- The levels in (37) are not equispaced though it appears that, whenever $\Lambda p \gg 1$, the entropy spectrum can be approximated with a formula granting equispaced levels. This limit can be achieved in two ways; in the first we consider great integer values for the quantum number $p$ whereas, in the second case, we deal with the limit $\Lambda \gg 1$. In this latter scenario, it is interesting to remark that the spectrum tends to its asymptotically flat counterpart (28).

- the spectrum (37) is consistent with the AdS-Schwarzschild black hole namely if we consider a vanishing electric charge, the electric sector contribution cancels i.e. $S(M)=2 \pi n+\pi$. This is a universal result proper of any two dimensional dilatonic gravity and thus also of the spherically symmetric reduced scenario of a Schwarzschild black hole, independently from the value of the cosmological constant [15] 16]. 
- if we interpret the quantum number $n$ as a measure of the excitation of the AdS Reissner-Nordstrøm black hole above extremality, we find, setting $n=0$ in (37) and independently from the charged sector, a non zero entropy which can be interpreted as the symptom of vacuum fluctuations. This result coincide with that achieved in the asymptotically flat counterpart, namely, within this quantization scheme the extremal configuration does not lie in the spectrum. To a certain extent this result confirms the statement (see [18] and [33]) according to which a third law of thermodynamic for black holes can be formulated: it is impossible that a non extremal black hole decays in an extremal configuration in a finite sequence of physical processes.

- bearing in mind that the adiabatic invariant for a non neutral black hole is the difference between the area and its extremal value, we can infer from a direct inspection of (34) the value of $a_{0}$ in (11) i.e. $8 \pi l_{p}^{2}$ as in the asymptotically flat Reissner-Nordstrøm scenario. The reader should also take into account that the constant term in (34) was not considered in Bekenstein ansatz though it is a natural feature both in the reduced phase space approach and in the axiomatic one discussed in the appendix.

- within the framework of Bekenstein formulation of black hole spectroscopy, a key result lies in the equivalence for a charged black hole between the reduced phase space approach and the axiomatic one, the latter reviewed in the appendix. As shown in 34 it is possible to start from the Hamiltonian formulation on the reduced phase space and to construct, as in the axiomatic scenario, an algebra of operators in terms of the variables $\left(M, \Pi_{M}, Q, \Pi_{Q}\right)$. Within this framework the two above mentioned approaches are identical with a due exception: the area operator considered by Bekenstein is equivalent in the reduced phase space approach to the the difference between the area of the event horizon and the area in the extremal configuration. Furthermore it appears that the eigenvalue which is obtained in [34 i.e. (34) is different from the Bekenstein one (43) up to a sign in the lowest eigenvalue; to the best of our knowledge such discrepancy has no clear explanation and it should be investigated carefully. Nonetheless the reasoning of 34 can be smoothly extended to our scenario and also, in an extremal AdS Reissner-Nordstrøm black hole, the equivalence between the axiomatic and the reduced phase space methods can be claimed.

\section{Conclusions}

In this paper we have explicitly constructed the area spectrum (37) for an AdS Reissner-Nordstrøm black hole and, in particular, we have shown that the contribution from the extremal part and the charged sector breaks the equispacing between the eigenvalues of the area operator. This result is ultimately true only in the Euclidean regime as in the asymptotically flat scenario, but we are 
not aware of a universally accepted derivation of area spectra in a Lorentzian background. Nonetheless we believe that the result could be highly interesting if considered as a starting point for future line of researches. In particular, besides addressing the natural question of the de Sitter counterpart of our analysis, the next direct step consists of considering a rotating black hole. In the asymptotically flat case, the Kerr-Newman solution has been studied in detail in [8, the main a priori difficulty lying in the non separability of the charge and the spin sector. This implies that this scenario cannot be seen as a simple extension of the charged non rotating case [5] or of the neutral rotating case [9]; a similar consideration could be applied to the results of this paper and thus the analysis of the AdS-Kerr-Newman black hole deserves careful considerations.

A further interesting application of the reduced phase space approach to black hole spectroscopy has been developed in section $\mathrm{V}$ of 35, where it has been shown that a direct consequence of an equispaced area/entropy spectrum is a markedly discrete spectrum for the Hawking radiation in the considered background. On the opposite, when the area spectrum in not equispaced (but discrete) such as in the three dimensional BTZ black hole or in the five dimensional rotating black hole, the corresponding spectrum for the Hawking radiation is quasi-continuum. Thus, in our scenario, we can slavishly repeat the analysis from the above cited paper starting from the first law of thermodynamics for a charged non rotating black hole

$$
\delta M=T_{H} \delta S+\phi \delta Q
$$

where $T_{H}$ is the Hawking temperature and $\phi$ is the electrostatic potential which on the horizon can be set to $\phi=\frac{Q}{r_{+}}$. If we take into account that $|\delta M|$ is proportional to the lowest frequency $\omega_{0}$ emitted in the Hawking radiation for the transition of a black hole from an excited level to its next lower state, the above equation becomes

$$
\delta \omega_{0}=\pi T_{H}\left[\delta n+\left(1+\frac{1}{2 \Lambda p \sqrt{1+4 \Lambda^{2} p^{2}}}\right) \delta p\right]+\frac{e^{2} p}{r_{+}} \delta p,
$$

where we have set $Q=p e$ and we have used the entropy spectrum formula (37). A direct inspection shows that the AdS Reissner-Nordström black hole shares the same feature than the black hole considered in [35] i.e. if we consider a variation $\delta n=1$ but $\delta p=0$, the spectrum appears to be equispaced. This is not surprising since it is a direct consequence of the reduced phase space approach which ultimately grants us that the spectrum of the black hole entropy minus the contribution from the extremal configuration is that of an harmonic oscillator. Conversely, if we choose $\delta n=0$ but $\delta p=1$, the spectrum is not equispaced and if we consider macroscopic black holes and large quantum numbers (i.e. $n, p \gg 1$ and $r_{+} \gg e^{2} p$ ), (38) tends to 0 for $p \rightarrow \infty$ i.e. the spectrum for the Hawking radiation appears to be quasi-continuum. It is fair to admit that this is more a preliminary remark more than a definitive claim; hence it would be interesting to repeat the above analysis in different asymptotically AdS backgrounds since 
the physical origin of the above behaviour apparently lies in the presence of the cosmological constant ${ }^{1}$.

On a broader setting, as briefly stated in the introduction, the results from this paper should be compared with different methods of area quantization the most notables arising from loop quantum gravity [11] and from the interpretation of the discreteness of the spectrum as an absorption of quasi normal mode excitations [13, 12] (see also [36] for an application to asymptotically flat extremal Reissner-Nordstrøm black holes and 37. for an application to BTZ black holes). In particular the area spectrum calculated in this last approach is completely different from the one calculated with the Hamiltonian framework we have introduced in the previous sections. In the asymptotically flat scenario there is no deep explanation for this discrepancy and, thus, we believe that the results in this paper could be, from the Hamiltonian point view, a starting point to extend the comparison between the above two mentioned methods in a wider class of spacetimes though the analysis of area spectra from quasi-normal modes in an AdS background is still far from complete (for a calculation of quasi normal modes for the AdS Reissner-Nordstrøm black hole see [38]).

Moreover it would be of great interest to analyze the results in this paper from the point of view of an holographic reconstruction of black hole physics. This specific point of view has not been pursued in the previous analysis of black holes spectra mainly due to the lack of a complete construction of an holographic correspondence in an asymptotically flat background. Conversely, in this paper we dealt with an asymptotically AdS spacetime which is the natural setting for the (asymptotically) AdS/CFT correspondence and it is important to remember that the black hole entropy is related to a conformal group or, more properly, to the associated Virasoro algebra by means of the Cardy formula. The usual rationale consists on constructing a conformal group on the horizon itself though it has been shown that this is not a unique point of view and it is also possible to deduce the statistical black hole entropy starting from the conformal asymptotic symmetry group as in [39] where the entropy of a two dimensional black hole ${ }^{2}$ is calculated in the framework of the $A d S_{2} / C F T_{1}$ correspondence. Thus, from a broader point of view, our hope is that the area operator for an AdS black hole could always be interpreted as a suitable dual quantum mechanical operator in the boundary conformal field theory and that the analysis of the spectrum of such an operator could be the same or at least related with the one proposed in our analysis by means of a semiclassical argument. To the best of our knowledge, such line of research has not been pursued yet and we are currently investigating it.

\footnotetext{
${ }^{1}$ In the AdS-Schwarzschild scenario, the spectrum for the Hawking radiation is clearly equispaced since the only contribution to the entropy comes from the "harmonic oscillator" as we have underlined at the end of the previous section

${ }^{2}$ The reader should bear in mind that, as it was discussed in the previous sections, a four dimensional spherically symmetric black hole can be equivalently described by means of a two dimensional black hole and of a dilaton field by means of a dimensional reduction procedure.
} 


\section{A The axiomatic approach}

The first approach to black hole area quantization, often referred to as the algebraic or axiomatic formulation, has been discussed in 40, 41, 42, 43. The underlying rationale consists of introducing a suitable set of operators defining a closed algebra by means of their commutation relations. The starting point lies in the intuitive assumption that it exists a suitable Fock space $\mathcal{F}$ whose elements $|n q j m s\rangle$ describe a one-black hole state which is characterized by the eigenvalues of the following set of operators $(\hbar=1)$ :

- an area operator $\hat{A}$ such that $\hat{A}|n q j m s\rangle=a_{n}(q, j, m)|n q j m s\rangle, n \in \mathbb{N}$, $a_{0} \doteq 0$ and $a_{n+1}>a_{n}$,

- a charge operator $\hat{q}$ such that $\hat{q}|n q j m s\rangle=q e|n q j m s\rangle$ where $q \in \mathbb{Z}$.

- the usual angular momentum operators $\hat{J}^{2}, \hat{J}_{e_{i}}$ such that $\hat{J}^{2}|n q j m s\rangle=$ $j(j+1)|n q j m s\rangle$ with $j$ a positive integer or semi-integer. $\hat{J}_{e_{i}}$ is the projection of $\hat{J}$ along a fixed direction $e_{i}$ and $\hat{J}_{e_{i}}|n q j m s\rangle=m|n q j m s\rangle$ with $m=-j, \ldots ., j$ for a fixed $j$.

The last quantum number, $s$, distinguishes between elements in $\mathcal{F}$ with the same values of $(n q j m)$ and thus it runs from 1 to the degeneracy $g_{n}$ of the state $|n q j m s\rangle$. It is possible to further characterize a state with fixed value of $s$ introducing a suitable operator which admits $s$ itself as an eigenvalue [8]. Unfortunately such approach requires that the equispacing of the spectrum of the area operator is a priori imposed, but this is a strong request which may not be satisfied in several physically interesting scenarios. Conversely, we will stick to the approach, first introduced in [40] and in [42, where it is assumed that it exists an operator $\hat{R}_{n q j m s}$ such that

$$
\hat{R}_{n q j m s}|v a c\rangle=|n q j m s\rangle,
$$

where $|v a c\rangle \in \mathcal{F}$ is the unique state $|00000\rangle$.

If we also introduce the identity $\hat{I}$, the set of operators $\hat{A}, \hat{q}, \hat{J}^{2}, \hat{J}_{e_{i}}, \hat{I}$ together with $\hat{R}_{n q j m s}$ is required to form a closed and linear algebra; besides the natural property of closure, the linearity is a strong assumption justified only by the physical prescription that $\hat{A}$ forms an additive quantity whenever several black holes are taken into account. Following the calculations of [40, 41, we introduce for simplicity the usual operators $\hat{J}_{ \pm}=\hat{J}_{e_{l}} \pm i \hat{J}_{e_{k}}$ where $\left(e_{i}, e_{k}, e_{l}\right)$ form an orthonormal base in $\mathbb{R}^{3}$ and where $\hat{J}^{2}=\frac{1}{2}\left(\hat{J}_{+} \hat{J}_{-}+\hat{J}_{-} \hat{J}_{+}\right)+\hat{J}_{e_{i}}^{2}$; the algebra defining commutation relations are:

$$
\begin{gathered}
{\left[\hat{q}, \hat{R}_{n q j m s}\right]=q e \hat{R}_{n q j m s}, \quad\left[\hat{J}_{e_{i}}, \hat{R}_{n q j m s}\right]=m \hat{R}_{n q j m s},} \\
{\left[\hat{J}_{ \pm}, \hat{R}_{n q j m s}\right]=\sqrt{j(j+1)-m(m \pm q)} \hat{R}_{n q j m \pm 1 s},} \\
{\left[\hat{A}, \hat{R}_{n q j m s}\right]=a_{n} \hat{R}_{n q j m s}+\delta_{q}^{0}\left[\delta_{j}^{0}\left(D_{n s} \hat{q}+E_{n s} \hat{A}\right)+\delta_{j}^{1} F_{n s} \hat{J}_{e_{i}}\right],}
\end{gathered}
$$


where $D_{n s}, E_{n s}, F_{n s}$ are arbitrary complex numbers for all $n, s$ and where all other commutators either are 0 or they assume the canonical expression as in quantum mechanics.

Starting from the above ansatz, it is possible to construct the area spectrum for a spherically symmetric black hole as in [40] and as in [41. Without entering into the details of Bekenstein calculations, let us nonetheless show the main results. The first step consists of noticing that the above commutation relations, in particular $\left[\hat{A}, J_{e_{i}}\right]=0$, grant us the rotational invariance for the area eigenvalues i.e. $a_{n}(q, j, m)=a_{n}(q, j)$. Moreover a similar argument excludes the dependence on the $j$ quantum number and, eventually, the one-black hole area operator depends only on the charge $q$; thus a long and tedious calculation shows that the eigenvalues $a_{n}$ must satisfy, for a neutral black hole, the identity

$$
a_{n}(0)=n a_{1}(0),
$$

whereas, for a charged black hole,

$$
a_{n}(q)=\left(n-\frac{1}{2}\right) a_{1}(0) .
$$

Thus $a_{1}(0)$ plays the role of $a_{0}$ in (11) and, whenever the black hole is charged, (43) shows that there is also a constant contribution which was not present in (11). Let us conclude with two important remarks: from one side the above discussed approach does not provide any means to explicitly calculate a precise

value for $a_{1}(0)$; from the other side either (42) either (43) grant us that the area spectrum for a black hole is uniformly spaced without any need to assume a priori such behaviour as in 8 .

\section{Acknowledgments}

The authors wish to thank Mauro Carfora for useful discussions and the referee for pointing out the relation between Hawking radiation and black hole spectroscopy as developed in Ref. 35. The work of C.D. is supported by a grant from the Department of Theoretical and Nuclear Physics - University of Pavia.

\section{References}

[1] J. D. Bekenstein and V. F. Mukhanov, "Spectroscopy of the quantum black hole," Phys. Lett. B 360 (1995) 7 arXiv:gr-qc/9505012,

[2] J. D. Bekenstein, "Quantum black holes as atoms," arXiv:gr-qc/9710076

[3] J. D. Bekenstein, "The Quantum Mass Spectrum Of The Kerr Black Hole," Lett. Nuovo Cim. 11 (1974) 467,

[4] A. E. Mayo, "Evidence for the adiabatic invariance of the black hole horizon area," Phys. Rev. D 58 (1998) 104007 arXiv:gr-qc/9805047. 
[5] A. Barvinsky, S. Das and G. Kunstatter, "Spectrum of Charged Black Holes - The Big Fix Mechanism Revisited," Class. Quant. Grav. 18, 4845 (2001) arXiv:gr-qc/0012066.

[6] S. Das, P. Ramadevi and U. A. Yajnik, "Black hole area quantization," Mod. Phys. Lett. A 17, 993 (2002) arXiv:hep-th/0202076.

[7] C. Vaz and L. Witten, "Mass quantization of the Schwarzschild black hole," Phys. Rev. D 60, 024009 (1999) arXiv:gr-qc/9811062.

[8] G. Gour and A. J. M. Medved, "Quantum spectrum for a Kerr-Newman black hole," Class. Quant. Grav. 20, 1661 (2003) arXiv:gr-qc/0212021.

[9] G. Gour and A. J. M. Medved, "Kerr black hole as a quantum rotator," Class. Quant. Grav. 20, 2261 (2003) arXiv:gr-qc/0211089.

[10] O. Aharony, S. S. Gubser, J. M. Maldacena, H. Ooguri and Y. Oz, "Large $N$ field theories, string theory and gravity," Phys. Rept. 323 (2000) 183 arXiv:hep-th/9905111.

[11] C. Rovelli and L. Smolin, "Discreteness of area and volume in quantum gravity," Nucl. Phys. B 442 (1995) 593 [Erratum-ibid. B 456 (1995) 753] arXiv:gr-qc/9411005.

[12] S. Hod, "Bohr's correspondence principle and the area spectrum of quantum black holes," Phys. Rev. Lett. 81 (1998) 4293 arXiv:gr-qc/9812002.

[13] O. Dreyer, "Quasinormal modes, the area spectrum, and black hole entropy," Phys. Rev. Lett. 90 (2003) 081301 arXiv:gr-qc/0211076.

[14] M. R. Setare, "Quasinormal modes, reduced phase space and area spectrum of black holes," arXiv:hep-th/0508222

[15] A. Barvinsky and G. Kunstatter, "Mass spectrum for black holes in generic 2-D dilaton gravity," arXiv:gr-qc/9607030

[16] A. Barvinsky and G. Kunstatter, "Exact physical black hole states in generic 2-D dilaton gravity," Phys. Lett. B 389, 231 (1996) arXiv:hep-th/9606134,

[17] A. Barvinsky, S. Das and G. Kunstatter, "Quantum mechanics of charged black holes," Phys. Lett. B 517, 415 (2001) arXiv:hep-th/0102061.

[18] A. Barvinsky, S. Das and G. Kunstatter, "Discrete spectra of charged black holes," Found. Phys. 32, 1851 (2002) arXiv:hep-th/0209039.

[19] R. M. Wald "General Relativity", (1984) The University of Chicago Press.

[20] D. Grumiller, W. Kummer and D. V. Vassilevich, "Dilaton gravity in two dimensions," Phys. Rept. 369 (2002) 327 arXiv:hep-th/0204253. 
[21] S. Nojiri and S. D. Odintsov, "Quantum dilatonic gravity in $d=2,4$ and 5 dimensions," Int. J. Mod. Phys. A 16 (2001) 1015 arXiv:hep-th/0009202.

[22] S. Nojiri and S. D. Odintsov, "Anomaly induced effective actions in even dimensions and reliability of s-wave approximation," Phys. Lett. B 463 (1999) 57 arXiv:hep-th/9904146.

[23] A. J. M. Medved and G. Kunstatter, "Hamiltonian thermodynamics of charged black holes," Phys. Rev. D 59, 104005 (1999) arXiv:hep-th/9811052.

[24] D. Louis-Martinez and G. Kunstatter, "Two-dimensional dilaton gravity coupled to an Abelian gauged field," Phys. Rev. D 52 (1995) 3494 [arXiv:grqc/950301

[25] D. Louis-Martinez and G. Kunstatter, "On Birckhoff's theorem in 2-D dilaton gravity,” Phys. Rev. D 49 (1994) 5227.

[26] P. A. M. Dirac, Lectures on Quantum Mechanics (1964) Yeshiva University, New York

[27] D. Louis-Martinez, J. Gegenberg and G. Kunstatter, "Exact Dirac quantization of all 2-D dilaton gravity theories," Phys. Lett. B 321 (1994) 193 arXiv:gr-qc/9309018.

[28] J. Gegenberg, G. Kunstatter and D. Louis-Martinez, "Observables for two-dimensional black holes," Phys. Rev. D 51, 1781 (1995). arXiv:gr-qc/9408015.

[29] C. Kiefer and J. Louko, "Hamiltonian evolution and quantization for extremal black holes," Annalen Phys. 8, 67 (1999) arXiv:gr-qc/9809005.

[30] B. Carter, "Black hole equilibrium states" in Les Astres Occlus edited by C. DeWitt and B. S. De Witt, (1972) Gordon and Breach Science Publisher,

[31] D. Kramer, H. Stephani, E. Herlt and M. MacCallum in "Exact Solutions of Einstein's Field Equations" edited by E. Schmutzer (1980) Cambridge Univeristy Press,

[32] J. Louko and S. N. Winters-Hilt, "Hamiltonian thermodynamics of the Reissner-Nordström-anti-deSitter black hole," Phys. Rev. D 54, 2647 (1996) arXiv:gr-qc/9602003.

[33] S. Das, A. Dasgupta and P. Ramadevi, "Can extremal black holes have nonzero entropy?," Mod. Phys. Lett. A 12 (1997) 3067 arXiv:hep-th/9608162.

[34] S. Das, P. Ramadevi, U. A. Yajnik and A. Sule, "Black hole area quantization. II: Charged black holes," Phys. Lett. B 565, 201 (2003) arXiv:hep-th/0207169, 
[35] S. Das, H. Mukhopadhyay and P. Ramadevi, "Spectrum of rotating black holes and its implications for Hawking radiation," Class. Quant. Grav. 22 (2005) 453 arXiv:hep-th/0407151.

[36] M. R. Setare, "Area spectrum of extremal Reissner-Nordstroem black holes from quasi-normal modes," Phys. Rev. D 69 (2004) 044016 arXiv:hep-th/0312061.

[37] M. R. Setare, "Non-rotating BTZ black hole area spectrum from quasi-normal modes," Class. Quant. Grav. 21 (2004) 1453 arXiv:hep-th/0311221.

[38] B. Wang, C. Y. Lin and E. Abdalla, "Quasinormal modes of ReissnerNordstroem anti-de Sitter black holes," Phys. Lett. B 481 (2000) 79 arXiv:hep-th/0003295.

[39] M. Cadoni and S. Mignemi, "Asymptotic symmetries of AdS(2) and conformal group in $d=1$," Nucl. Phys. B 557 (1999) 165 arXiv:hep-th/9902040.

[40] J. D. Bekenstein, "The case for discrete energy levels of a black hole," Int. J. Mod. Phys. A 17S1 (2002) 21 arXiv:hep-th/0107045.

[41] J. D. Bekenstein, "Quantum information and quantum black holes," arXiv:gr-qc/0107049

[42] J. D. Bekenstein and G. Gour, "Building blocks of a black hole," Phys. Rev. D 66 (2002) 024005 arXiv:gr-qc/0202034.

[43] G. Gour, "Quantum mechanics of a black hole," Phys. Rev. D 61 (2000) 124007 arXiv:gr-qc/9906116. 